\title{
Kinetics of cluster growth in expanding rare-gas jet
}

\author{
M. A. Ratner \\ B. I. Verkin Institute for Low Temperature Physics and Engineering, National Academy of Science of Ukraine, \\ 47 Lenin Ave., 310164 Kharkov, Ukraine, \\ E-mail: ratner@ilt.kharkov.ua \\ Received November 5, 1998
}

\begin{abstract}
The growth of clusters in an overcooled atomic jet is calculated with the simultaneous allowance for two processes: the condensation of supersaturated gas and the cluster-cluster aggregation. These processes are consistently described within a quasi-equilibrium approach for large enough clusters, while their nucleation stage is allowed for phenomenologically by the use of a fitting parameter. The theory quantitatively describes the experimental dependence of the mean cluster size on the initial gas temperature within a broad range of sizes. The mean size of clusters, their distribution over sizes and the contribution of clusters to the total mass of the jet are calculated versus the distance from the gas ejection point (nozzle entry). It is shown that these characteristics are mainly determined by the initial gas entropy which is equivalent to the empirical law of corresponding jets. The size distribution function has a halfwidth exceeding the mean cluster size and is close (on a relative scale) to an universal curve. The broad size distribution of clusters should be taken into account when analyzing spectroscopic data on the size dependence of electronic processes in a cluster.
\end{abstract}

PACS: 36.40.-c

\section{Introduction}

Experimental study of atomic clusters provides an alluring possibility of tracing the evolution of energy spectrum, relaxation processes and atomic structure when varying the size of an atomic aggregate from several atoms to a macroscopic number. Of particular interest are rare-gas clusters due to their distinctive feature - the simplest atomic and electronic structure that is due to a weak van der Waals binding. During recent years, the spectroscopy of rare-gas clusters has been intensively developed due to great progress in the supersonic gas-jet technique [1-10]. It has been possible to trace essential size variations in luminescence, absorption and energy spectra [2-8] as well as in the growth mechanism [9].

The aim of spectroscopic and electronographic studies of clusters is to obtain the dependences of electronic and atomic structure on cluster size. In fact, however, experiment does not directly give a physical quantity of interest as a function of cluster size. This quantity is averaged over cluster sizes with the weight of the size distribution of clusters in the expanding jet. Due to the large width in the size distribution function, the size dependence of the quantity or phenomenon under investigation is noticeably smeared.

To infer a more precise information from experimental data, it is necessary to know the size distribution function of clusters growing in the expanding jet.

The size distribution of clusters is formed in the course of their growth which is realized simultaneously in two ways: by condensation of the overcooled gas on the cluster surface and by clustercluster aggregation. Both mechanisms make comparable contributions to the formation of the distribution function and should be considered simultaneously.

There is a large number of theoretical works concerned with cluster growth in an expanding jet (e.g., Refs. [10-20]), but the size distribution function had not been calculated in any of them. In previous literature the condensation and aggregation mechanisms of cluster growth were considered separately.

Some thermodynamical and nonequilibrium aspects of the condensation mechanism (in particular, atom-cluster collisions and desorption of atoms from a cluster) were investigated in Refs. [10-13]. In Ref. [14] the kinetics of cluster growth was 
calculated by taking the condensation mechanism into account. The calculations were performed on the basis of the drop model of a cluster and classical nucleation with allowance for the dependence of the surface tension coefficient on the drop radius. As was shown in Ref. [14], the drop model, fitted with the real process of cluster growth by the use of free parameters, describes some important experimental regularities of cluster growth. However, such an approach does not give the size distribution of the clusters.

The mechanism of cluster-cluster aggregation has been studied in many works e.g., in Refs. [15-20]. The papers [15-17] were concerned mainly with combinatorial aspects of the aggregation process. In Refs. [18-20] the process of cluster-cluster collision was studied on a microscopic level and the cross section of fusion was considered for charged metal clusters with allowance for their structure.

The purpose of the present work consists in the calculation of the cluster growth kinetics, involving both the mentioned growth processes, which is necessary to obtain the size distribution function of clusters in an expanding jet.

As follows from the cited works [10-20], each of the above processes of cluster growth has a highly complicated nature even if considered separately. In order to calculate the joint process of cluster growth within a reasonable constructive approach, some simplifying assumptions are used below. In particular, although the cluster growth is an essentially nonequilibrium process, it is long enough that thermodynamic equilibrium is practically achieved within a separate cluster as well as in the gas of atoms and clusters considered as structureless particles with translational degrees of freedom. (But intrinsic degrees of freedom of clusters are not in equilibrium with gas, and it is just the lack of this equilibrium which causes the condensation of gas on clusters).

The nucleation stage of cluster growth is taken into account in a phenomenological way, so that the consistent consideration is restricted to a region of large enough clusters. In this region shell effects, although generally important [18,19], can not be taken into account. A nonmonotonic size dependence of bond energy, originated by shell effects, is inessential for the growth of a large cluster due to the large number of shells subsequently filled.

\section{Condensation of overcooled gas on the cluster surface}

Let us consider the nonequilibrium process of gas condensation on the surface of a cluster with the intrinsic temperature $T_{\mathrm{cl}}$. An atom-cluster collision results in their merging in the cluster temperature region

$$
T_{\mathrm{cl}}<T_{c}\left(N, S_{g}\right)
$$

where the merging is attended by an increase in the total entropy of the nonequilibrium system. The critical temperature of a cluster, $T_{c}$, is defined by the condition that the total entropy of the system does not change as a gas atom joins to the cluster. $T_{c}$ is a function of the cluster size $N$ (the number of atoms) and the gas entropy $S_{g}$. It is assumed for simplicity that every collision of an atom with a cluster of size $N$ results in their merging with unit probability if the inequality (1) is fulfilled and with zero probability in the opposite case.

Based on this premise, a quasi-stationary approach is introduced: during the process of cluster growth the temperature of every cluster is equal to its critical temperature $T_{c}\left(N, S_{q}\right)$ which varies with cluster size and gas state. If cluster temperature becomes lower than $T_{c}\left(N, S_{q}\right)$, i. e. the joining of an atom to the cluster is attended by an increase of the total entropy, then gas condensation on the cluster surface increases cluster temperature until it achieves $T_{c}$. This process goes very fast since every atom-cluster collision, attended by merging, enhances intrinsic cluster energy by the atom-cluster binding energy $U$ and cluster temperature by $U / 3 N$. Thus, the number of atom-cluster collisions, required to adjust cluster temperature to $T_{C}$, is of the order of $N T / U<N<<v$ where $v$ is the total number of cluster-atom collisions during the growth process. If the cluster temperature becomes higher than $T_{c}\left(N, S_{q}\right)$, then the desorption of atoms from the cluster surface becomes thermodynamically favorable and results in a fast decrease of the cluster temperature down to $T_{c}$.

The critical temperature of a cluster, $T_{c}\left(N, S_{g}\right)$, is defined from the condition that the augmentation of the cluster entropy, $S_{\mathrm{cl}}$, when joining one atom equals by magnitude the decrease of the gas entropy, $S_{g}$, when removing one atom:

$$
\begin{gathered}
S_{g}\left(T_{g}, V, N_{g}\right)-S_{g}\left(T_{g}, V, N_{g}-1\right)= \\
=S_{\mathrm{cl}}\left(\tilde{T}_{c}, N+1\right)-S_{\mathrm{cl}}\left(T_{c}, N\right)
\end{gathered}
$$

where $T_{g}, N_{g}$ and $V$ are the temperature, the number of atoms and the volume of the gas. $\tilde{T}_{c}$ is the temperature of the cluster immediately after joining one atom if the initial temperature of the cluster was equal to $T_{c} . \tilde{T}_{c}$ is defined by the equation 


$$
E_{\mathrm{cl}}\left(\tilde{T}_{c}, N+1\right)-E_{\mathrm{cl}}\left(T_{c}, N\right)=w[Z(N+1)-Z(N)]
$$

where $E_{\mathrm{cl}}$ is the intrinsic energy of a cluster, $w$ is the energy of one interatomic bond and $Z(N)$ is the total number of bindings between adjacent atoms in the $N$-atomic cluster. The $w$ value is assumed to be independent of the cluster temperature and size. This assumption means that the interatomic distances in a cluster are equal for all pairs of adjacent atoms and independent of cluster temperature (the latter is justified by a weak thermal expansion at low temperature). In addition, only the interaction between adjacent atoms is allowed for; this is justified by a fast decrease of the van der Waals interaction energy with distance.

In order to find the critical temperature, $T_{c}$, from the Eqs. (2), (3), it is necessary to substitute into them the expressions for thermodynamic functions of the gas and the cluster. The entropy of ideal single-atomic gas can be written in the well-known form

$$
\begin{gathered}
S_{g}=N_{g} s_{g}, s_{g}=\ln \left(e V / N_{g}\right)+c_{V} \ln \left(T_{g} e\right) \\
(e=\exp (1))
\end{gathered}
$$

where $s_{g}$ is gas entropy per atom; $N_{g}$ is the number of atoms in the gas; $V$ is gas volume and $c_{V}$ is the heat capacity of the gas per atom at a constant volume. Here and below the temperature is expressed in energy units, so that $c_{V}=3 / 2$ for a single-atomic gas.

Since the structure of an arbitrary-size cluster is unknown, it is reasonable to write down its thermodynamic functions within the Debye model. However, it is necessary to take account of the discreteness of the energy spectrum which can manifest itself at low temperatures for not very large clusters. For that purpose the cluster of the size $N=L^{3}$ is presented in the form of a cube with the lattice constant $a$ and the edge containing $L$ atoms (for definiteness $L$ is even). The phonon wavevector $\mathbf{K}$ takes up the values

$$
\mathbf{K}=(i-1 / 2, j-1 / 2, k-1 / 2)(\pi / L a)
$$

with $i, j, k=-L / 2, \ldots, L / 2$. Within the Debye model the vibration frequencies of the cluster are equal to $s_{l}|\mathbf{K}|$ for the longitudinal branch and to $s_{t}|\mathbf{K}|$ for the transverse branches. For a crystal with the van der Waals binding $s_{l} / s_{t}=\sqrt{3}$ [21]. Thus, the vibration spectrum of the cluster takes the form $\omega_{i, j, k}^{\alpha}=\frac{\hbar \omega_{D} q_{\alpha}}{T_{c l}} \frac{\sqrt{(i-1 / 2)^{2}+(j-1 / 2)^{2}+(k-1 / 2)^{2}}}{L}$.

Here $\omega_{D}$ is the Debye frequency, index $\alpha$ numbers vibrational branches. The coefficients $q_{1}=2.515$, $q_{2}=q_{3}=1.452$ fit the expression (5) with the Debye model for $N \rightarrow \infty$. Using the vibrational spectrum, one can write down the thermodynamical functions of the cluster:

$$
\begin{gathered}
E_{\mathrm{cl}}=N T_{\mathrm{cl}} \sum_{\substack{i, j, k=-L / 2 \\
\alpha=1,2,3}}^{L / 2}\left[\frac{\hbar \omega_{i, j, k}^{\alpha} / T_{\mathrm{cl}}}{1-\exp \left(-\hbar \omega_{i, j, k}^{\alpha} / T_{\mathrm{cl}}\right)}\right], \\
S_{\mathrm{cl}}=N \sum_{\substack{i, j, k=-L \\
\alpha=1,2,3}}^{L} \hbar\left\{\frac{\hbar \omega_{i, j, k}^{\alpha} / T_{\mathrm{cl}}}{1-\exp \left(-\hbar \omega_{i, j, k}^{\alpha} / T_{\mathrm{cl}}\right)}-\right. \\
-\ln \left[1-\exp \left(-\hbar \omega_{i, j, k}^{\alpha} / T_{\mathrm{cl}}\right)\right\} .
\end{gathered}
$$

The expressions $(6),(7)$ relate to a cluster with $N>>1$. In this case the contribution of the translational and rotational degrees of freedom to thermodynamic functions is negligibly small and is also weakly dependent on $N$. In Eqs. (6), (7) these degrees of freedom are omitted. Using interpolation nodes $N=L^{3}$, one can obtain $E_{\mathrm{cl}}$ and $S_{\mathrm{cl}}$ for an arbitrary $N$ via the interpolation of the quantities $E_{\mathrm{cl}} / N$ and $S_{\mathrm{cl}} / N$ weakly dependent on $N$.

The relations (2)-(7) define the critical temperature of a cluster, $T_{c}$, as a function of its size and the gas state. Figure 1 shows the dependence of $T_{c}$ on cluster size at various fixed values of gas entropy per atom $s_{g}$. For a given $N, T_{c}$ lowers with an increase of $s_{g}$, i.e., with raising gas temperature or lowering pressure. For a given $s_{g}, T_{c}$ increases with cluster size (which corresponds to the effect of surface tension on cluster growth within the drop model [14]) and achieves saturation for $N \gtrsim 1000$.

In Fig. 2 the gas-temperature dependence of $T_{c}$ is shown for $N=500$ and different concentrations of atoms in the gas. $T_{c}$ decreases with an increase of gas temperature due to the corresponding increase of gas entropy. This means that the cluster temperature (maintained close to $T_{c}$ ) heightens as the gas cools; such a relation between the temperatures of the gas and clusters illustrates a nonequilibrium character of the growth process in an expanding jet (due to a fast cooling of the gas, gas pressure much exceeds its equilibrium value). 


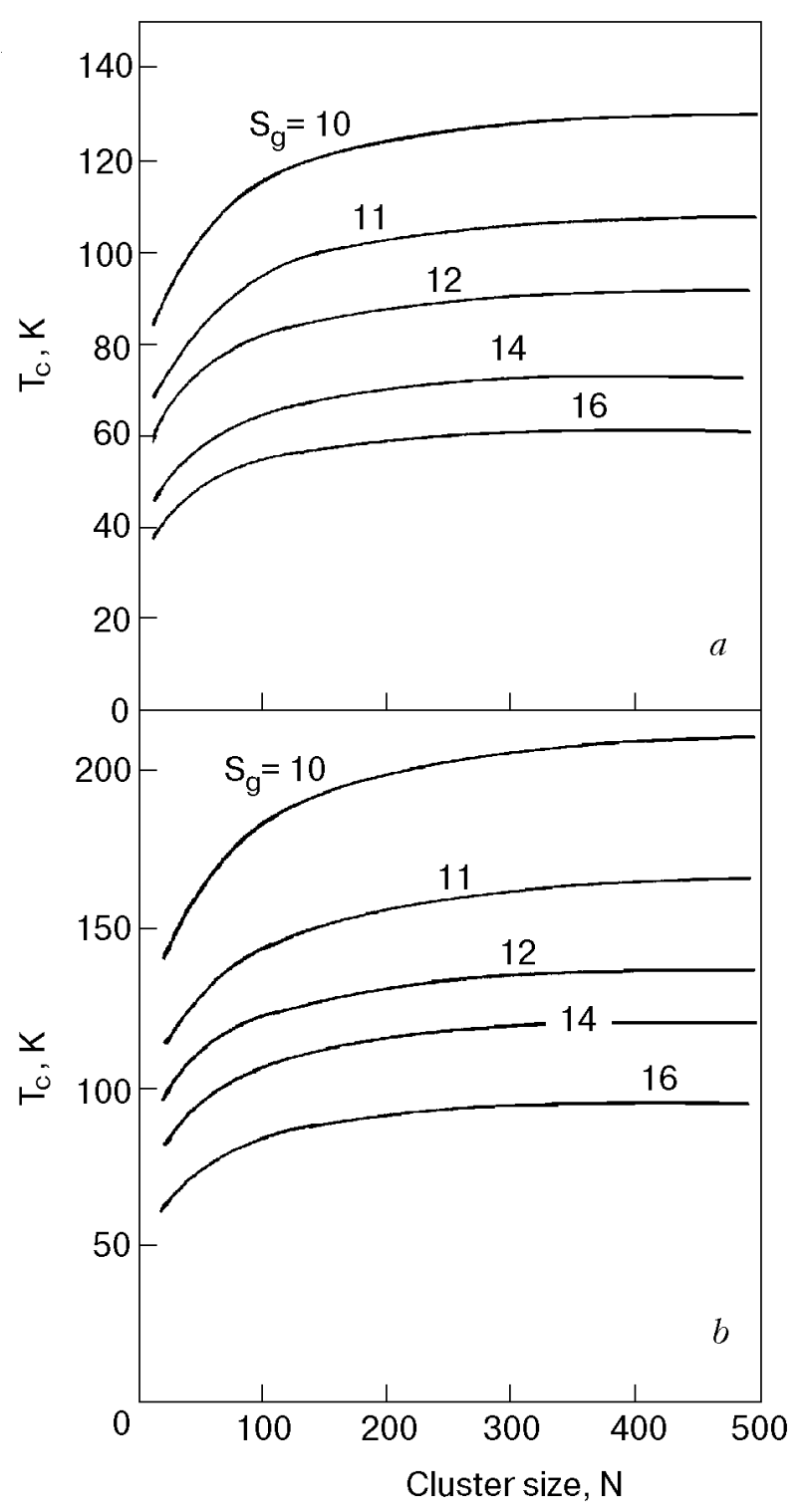

Fig. 1. Size dependence of the critical temperature, $T_{c}$, of a cluster for different fixed values of gas entropy per atom $s_{g}$ (indicated in the figure): argon $(a)$, krypton $(b)$.

A cluster grows if the difference $T_{c}-T_{g}$ (equal to the cluster-gas jump of temperature) is positive (contrary to an usual notion about the deposition of a gas on a cold surface). A positive gas-cluster jump of temperature in the course of cluster growth is necessary to provide an outflow of the heat, released due to gas condensation on the cluster surface, into gas. The difference $T_{c}-T_{g}$ is shown in Fig. 2 by a dashed line; the gas-temperature region of cluster growth is limited by the intersection point of the dashed line with the abscissa axis.

The cluster growth rate is proportional to the thermal flux $q(N)$ from the cluster surface to the gas, i. e., to the difference between cluster temperature (assumed to be equal to $T_{c}$ ) and the gas temperature:

$$
\begin{gathered}
\frac{d N}{d t}=\frac{q(N)}{d E_{\mathrm{cl}}\left[T_{c}\left(N, S_{g}\right), N\right] / d N}, \\
q(N)=\frac{3}{2}\left(T_{c}-T_{g}\right) \sigma(N, 1)\langle v\rangle N_{g} / V .
\end{gathered}
$$

Here $\langle v\rangle$ is the mean thermal velocity of gas atoms; $\sigma(N, 1)$ stands for collision section of an atom with the cluster of the size $N$. In the denominator of Eq. (8) the total derivative is meant. The expression for the collision section will be derived in Sec. 3 [see Eq. (17)].

\section{Cluster-cluster aggregation}

Although the cluster growth is an essentially nonequilibrium process, it is long enough that thermodynamic equilibrium is practically achieved not only within the gas and within every separate cluster but also within the subsystem of the translation degrees of freedom related to all the particles in the jet (this subsystem includes also the rotation motion of every cluster as a whole). The equilibrium in this subsystem is maintained in the course of the growth of a clusters since the total number, $v$, of collisions between the cluster and gas atoms much exceeds the number of atoms joined to the cluster i.e. $v>>N$. Thus, the gas-cluster exchange of energy (the exchange per one collision, $T_{g} / N$, multiplied by $v$ is large compared to the kinetic energy of a cluster as a whole $\left(\sim T_{q}\right)$. Taking this into account, we can describe distribution of clusters over translation motion velocities by the Boltzmann statistics with the temperature equal to $T_{g}$.

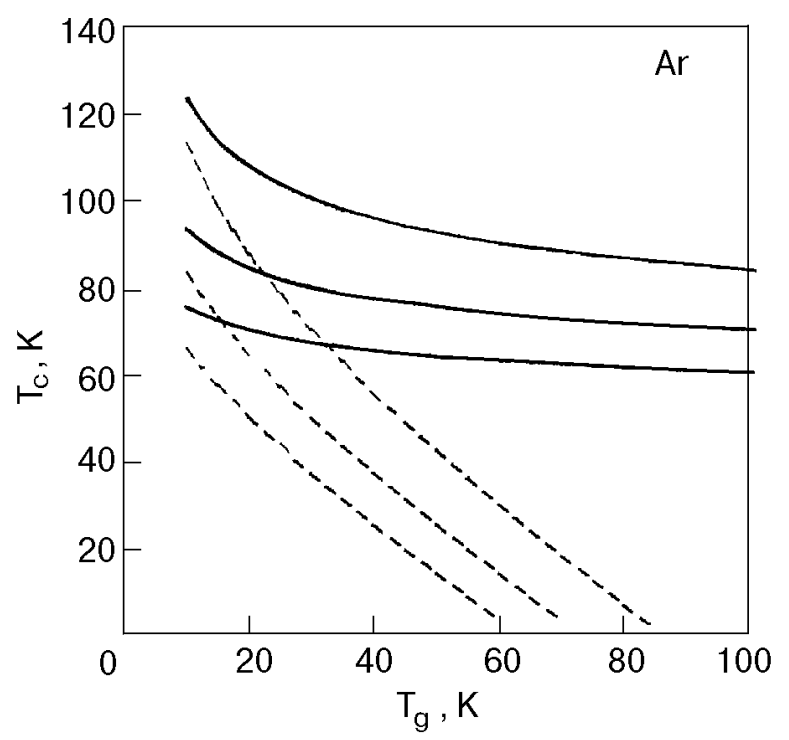

Fig. 2. Dependence of the critical temperature, $T_{c}$, of the argon cluster with $N=500$ on gas temperature, $T_{q}$, for the gas concentrations (from top to bottom): $10^{17}, 10^{16}$ and $10^{15} \mathrm{~cm}^{-3}$ solid lines). Dashed lines - the same for the difference $T_{c}-T_{g}$. 
The probability of the collision of two clusters, numbered by the subscripts $i$ and $j$, per unit time is

$$
\left\langle P_{i j}\right\rangle=\left\langle\mathbf{v}_{i}-\mathbf{v}_{j}\right\rangle\left\langle\sigma_{i j}\right\rangle / V=\left(8 T_{g} / \pi \mu_{i j}\right)^{1 / 2}\left\langle\sigma_{i j}\right\rangle / V .
$$

Here $\mathbf{v}_{i}$ is the velocity vector of the $i$-th cluster; $\sigma_{i j}$ is the collision section of the $i$-th and $j$-th clusters; $\langle\ldots\rangle$ denotes the Boltzmann averaging; $\mu_{i j}$ is the reduced mass of the clusters,

$$
\mu_{i j}=m_{\mathrm{at}} N_{i} N_{j} /\left(N_{i}+N_{j}\right)
$$

( $m_{\text {at }}$ is the atomic mass). In Eq. (10) $\sigma_{i j}$ is assumed to be weakly dependent on cluster velocities which permits one to take it out of the sign of averaging.

In order to derive $\sigma_{i j}$, let us substitute a cluster by the point particle with the same mass (the validity range of this approximation will be indicated below). Neutral clusters are attracted to each other by van der Waals forces, which create the potential

$$
U\left(r_{i j}\right)=-C_{i j} r_{i j}^{-6}, C_{i j}=C_{\mathrm{at}} N_{i} N_{j}
$$

with the atomic van der Waals constant $C_{\text {at }}$. It follows from the classical motion equation of two interacting particles that there exist two types of motion: the particles can either fall onto each other or move away from each other after drawing together to the minimal distance. These types of motions are separated by the critical aiming distance

$$
d_{i j}=\left(27 C_{i j} / 2 \mu\right)^{1 / 6}\left|\mathbf{v}_{i}-\mathbf{v}_{j}\right|^{-1 / 3} .
$$

The quantity (13), weakly dependent on velocities, can be averaged approximately over the Boltzmann distribution:

$$
\begin{aligned}
\left\langle d_{i j}\right\rangle \cong & \left(27 C_{i j} / 2 \mu\right)^{1 / 6}\left\langle\mathbf{v}_{i}^{2}+\mathbf{v}_{j}^{2}\right\rangle^{-1 / 6}= \\
& =\left(9 C_{\mathrm{at}} N_{i} N_{j} / 2 T_{g}\right)^{1 / 6} .
\end{aligned}
$$

The averaged collision section of point particles, representing the clusters, can be estimated as

$$
\left\langle\sigma_{\text {attr }}\left(N_{i}, N_{j}\right)\right\rangle \cong \pi\left\langle d_{i j}\right\rangle^{2} \cong \pi\left(9 C_{\mathrm{at}} N_{i} N_{j} / 2 T_{g}\right)^{1 / 3}
$$

(the subscript «attr» indicates that the collision of particles is caused but their van der Waals attraction).

The attraction section of cluster-cluster collisions (15) should be compared with the geometrical collision section

$$
\sigma_{\text {geom }}\left(N_{i}, N_{j}\right) \cong \pi l^{2}\left(N_{i}^{1 / 3}+N_{j}^{1 / 3}\right)^{2} / 4
$$

where $l$ is the interatomic distance in solid. The true collision section is

$$
\sigma\left(N_{i}, N_{j}\right)=\max \left\{\sigma_{\text {attr }}\left(N_{i}, N_{j}\right), \sigma_{\text {geom }}\left(N_{i}, N_{j}\right)\right\} .
$$

The ratio of the attractive and geometrical sections is

$$
\begin{gathered}
\sigma_{\mathrm{attr}}\left(N_{i}, N_{j}\right) / \sigma_{\text {geom }}\left(N_{i}, N_{j}\right) \cong \\
\cong\left(9 U_{\mathrm{mol}} / 2 T_{g}\right)^{1 / 3} 4\left(N_{i} N_{j}\right)^{1 / 3}\left(N_{i}^{1 / 3}+N_{j}^{1 / 3}\right)^{-2}
\end{gathered}
$$

where $U_{\mathrm{mol}}=C_{\mathrm{at}} l^{-6}$ stands for the bond energy of the two-atomic van der Waals molecule. For argon, krypton or xenon $U_{\mathrm{mol}}>T_{g}$, and for clusters of comparable sizes the ratio (18) exceeds unity. The collision section (15) will be used as applied to cluster-cluster aggregation by taking this into account.

However, for atom-cluster collisions the ratio (18) becomes less than unity starting from $N \sim 1000$. Therefore, the collision section $\sigma(N, 1)$ in the form (17) is substituted into the expression (9) for thermal flux.

Since the collision of two clusters is caused by their attraction, and the attraction energy at the collision moment much exceeds the initial kinetic energy of the colliding clusters in magnitude, it can be assumed that every collision of clusters results in their fusion. Indeed, if the final kinetic energy (slightly exceeding in magnitude the attraction energy at the collision moment) is partially transformed to heat, the rest of the kinetic energy becomes less than the magnitude of the attraction energy and is insufficient for the clusters to move away from each other.

The aggregation of the clusters with the sizes $N_{1}$ and $N_{2}$ and temperatures equal to their critical temperatures $T_{c 1}$ and $T_{c 2}$ results in the formation of the cluster with the size $N_{3}=N_{1}+N_{2}$ and temperature $T_{3}$. The difference between $T_{3}$ and $T_{c}\left(N_{3}, S_{g}\right)$ is found to be negligibly small so that the temperature of the formed cluster can be put equal to $T_{c}\left(N_{3}, S_{g}\right)$.

\section{Kinetics of cluster growth involving condensation and aggregation mechanisms}

The processes of cluster growth via gas condensation and cluster-cluster aggregation, considered in the previous sections, are determined by the gas state in the expanding jet as a function of time $t$. We trace the gas state within a small volume $V$ which moves together with the jet and contains a 
constant number of atoms, $N_{\text {tot }}$ (the part of them, denoted by $\xi N_{\text {tot }}$, has joined to clusters). The motion of the volume $V$ begins from the nozzle entry, which is a small hole through which the gas flows out from the reservoir to the vacuum through the nozzle. The gas state is described by the usual equation:

$$
P V=N_{g} T_{g}, N_{g}=N_{\text {tot }}(1-\xi), N_{\text {tot }}=\text { const }
$$

( $P$ is gas pressure; the number of clusters is negligibly small compared to the number of free atoms $\left.N_{\text {tot }}(1-\xi)\right)$. The adiabatic expansion of the jet is described by the known equations (see, e. g., [14]):

$$
\begin{gathered}
u S / V=\text { const, } \\
\left(N_{\text {tot }} m_{\mathrm{at}} / V\right) u \frac{d u}{d x}+\frac{d P}{d x}=0, \\
N_{g} m_{\mathrm{at}} c_{P}\left(T_{g}-T_{0}\right)+N_{\mathrm{tot}} m_{\mathrm{at}} u^{2} / 2=\int_{0} \sum_{t} q\left(N_{i}\right) d t .
\end{gathered}
$$

Here $x$ is the distance of the chosen elementary volume from the nozzle entry at the moment $t$; $u=d x / d t$ is the jet velocity at the point $x ; S$ is the cross section of the chosen volume at the point $x$ (the transverse nonuniformity of the jet is neglected); $c_{P}=5 / 2$ is the heat capacity of gas per atom at a constant pressure; $T_{0}$ is the temperature at the nozzle entry. The right-hand part of Eq. (22) is the total heat released due cluster formation (the sum is taken over all the clusters), the thermal flux $q\left(N_{i}\right)$ is given by Eq. (9). It is implied in Eqs. (21), (22) that the clusters are moving with the same velocity as the gas.

The equations (19)-(22) describe the gas state for a given distribution of clusters over sizes. For a given gas state, the time evolution of the size distribution of clusters is calculated with the simultaneous allowance for the condensation and aggregation mechanisms (Secs. 2 and 3). Thus, the selfconsistent variations of the gas and cluster subsystems is calculated.

Such approach is realized in the region of large sizes, where the thermodynamic consideration of Sec. 2 is valid, and must be complemented by the initial conditions which are formed on the stage of the formation of cluster nuclei in the jet. The microscopic consideration of the nucleation process is a highly complicated problem which is beyond the framework of the present work. Therefore, the formation of cluster nuclei is described in a phenomenological way on the basis of the following considerations.
The heat release due to the formation of nuclei does not make any noticeable contribution to the thermal balance of the jet. On the other hand (as was shown in Ref. [14] and by the present calculations), the duration of the nucleation stage is short compared with the total time of cluster growth in the jet. Therefore, the influence of nucleation stage on the further growth of clusters is determined by two parameters: the number of the nuclei formed, $n_{\text {nucl }}$, and the moment of the nucleation onset, $t_{\text {on }}$. The mean size of clusters decreases with increasing $n_{\text {nucl }}$ and $t_{\text {on }}$ (the earlier clusters arise and begin to grow the larger they become at a given time moment or at a given point $x$ ).

Within the phenomenological description, the nucleation onset $t_{\mathrm{on}}$ is determined by the critical temperature of nucleation, $T_{\text {nucl }}$, and is defined by the equation

$$
T_{g}\left(t_{\text {on }}\right)=T_{\text {nucl }}
$$

(here $T_{g}$ is the gas temperature in the elementary volume of the expanding atomic jet devoid of clusters). Without nucleation, gas temperature in the expanding jet would decrease below $T_{\text {nucl }}$; due to the heat release, accompanying the rise and subsequent growth of clusters, the gas temperature $T_{g}$ is maintained at the level $T_{\text {nucl }}$ until the moment $t_{\text {end }}$ when $T_{g}$ begins to heighten in the course of cluster growth. In the time interval $t_{\text {on }}<t<t_{\text {end }}$, the number of arising clusters is determined by the competition between nucleation and cluster growth. This competition is regulated by the initial cluster size, $N_{\text {init }}$, and cluster growth rate; $n_{\text {nucl }}$ decreases with an increase in every of these parameters. Since they are unknown, it is reasonable to choose each of them in such a way that the related errors partially compensate each other. Hence, $N_{\text {init }}$ is put equal to the minimal size, 4 , of a cluster with the 3-dimensional configuration of atoms. To compensate the corresponding error, the cluster growth rate, defined by Eqs. (8),(9), is somewhat overestimated: the critical temperature $T_{c}$ is taken for a cluster with $N=64$ (this is the minimal cluster size for which the thermodynamic consideration of Sec. 2 is certainly applicable).

The critical temperature of nucleation, $T_{\text {nucl }}$, depends on the gas pressure $P$. It is assumed that this dependence does not essentially differ in its character from the known relation between temperature and pressure for the gas-solid or gas-liquid equilibrium. So, the dependence of $T_{\text {nucl }}$ on gas pressure $P$ is written down in the form

$$
T_{\text {nucl }}=w \Delta Z / \ln \left(P^{*} / P\right) .
$$



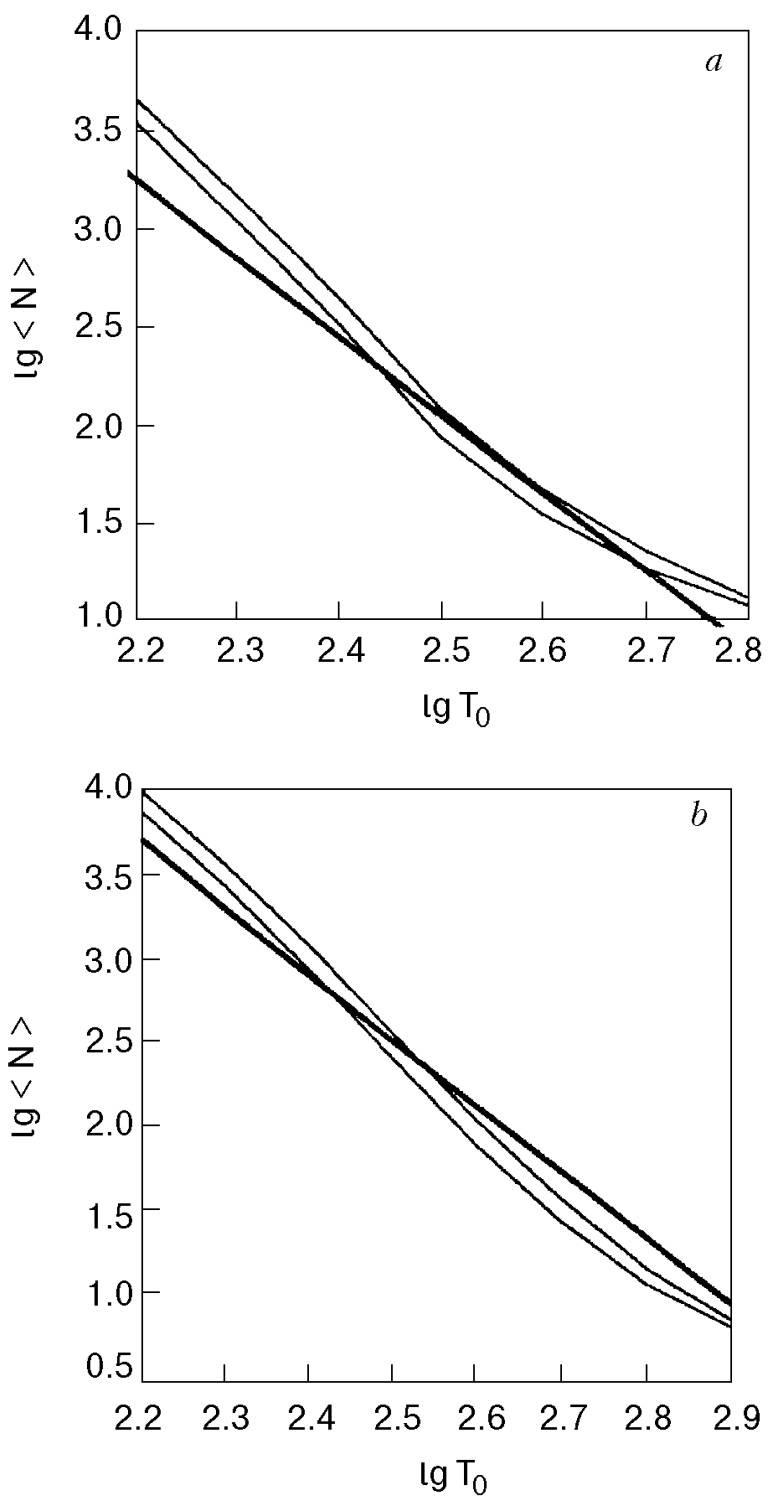

Fig. 3. Dependence of the mean cluster size on the initial gas temperature, $T_{0}$, at the $4.1 \mathrm{~cm}$ distance from the nozzle entry for argon $(a)$ and krypton $(b)$ (the initial pressure $P_{0}$ is constant and equal to $1 \mathrm{~atm}$ ). Thick lines - experimental data [14, 22]; thin lines - calculation results for the fitting parameter values (from top to bottom): $P^{*}=100 \mathrm{~atm}$ and $500 \mathrm{~atm}$ (the best fit) for argon; $P^{*}=10^{5} \mathrm{~atm}$ (the best fit) and $10^{6} \mathrm{~atm}$ for krypton.

Here $\Delta Z=3$ is the variation of the number of bonds when joining or removing one atom from the incipient cluster with $N=4$; $w$ is the energy per one bond. $P^{*}$ is a fitting parameter of the phenomenological description of nucleation and by no means cannot be identified with a true equilibrium parameter. Note that the nucleation process, in view of its complicated character, can hardly be described by the law of corresponding states; hence, this law is generally not met by Eq. (24) either.

The results below are related to the fitting parameter values $P^{*}=500 \mathrm{~atm}$ for argon and $10^{5} \mathrm{~atm}$ for krypton which provide the best agreement of the theory with the experimental data on the mean cluster size.

\section{Calculation results and comparison with experiment}

The calculation of the cluster growth kinetics in an expanding gas jet was carried out according to the above scheme, including condensation and aggregation processes, for argon and krypton. The following characteristics of a cluster jet have been calculated.

Every characteristic of clusters, that can be measured experimentally is determined by the distribution of the total mass of clusters over their sizes. This distribution function is defined as

$$
F(N)=d\left(\sum_{N_{i}<N} N_{i}\right) / d N
$$

The mean size of clusters in the jet is defined by means of the distribution function (25):

$$
\langle N\rangle=\frac{\int F(N) N d N}{\int F(N) d N}=\frac{\sum N_{i}^{2}}{\sum N_{i}} .
$$

The results below are related to a conical nozzle with entry diameter equal to $0.34 \mathrm{~mm}$ and apex angle $8.6^{\circ}$.

Figure 3 shows the mean size of clusters as a function of the gas temperature at the nozzle entry, $T_{0}$, for the constant pressure at the nozzle entry $P_{0}=1 \mathrm{~atm}$. The thick line denotes the experimental data measured at the distance $x=4.1 \mathrm{~cm}$ from the nozzle entry [14, 22] and the thin lines represent the corresponding calculation results related to different values of the fitting parameter $P^{*}$. As seen from the figure, in the case of the best fit $\left(P^{*}=500 \mathrm{~atm}\right.$ for argon and $10^{5}$ atm for krypton) the theoretical curve agrees with the experimental one with the accuracy up to the multiplier 1.3 to 1.8 , while the mean cluster size varies by two orders of magnitude. Note that the agreement between the theory and experiment takes place on the absolute scale ( $x$ is expressed in centimeters, $T_{0}$ in Kelvins and $\langle N\rangle$ in the number of atoms). This evidences for the validity of the theory based on the above-enumerated assumptions.

The calculation results, presented in Figs. 4 to 6 , are related to the case of the best fit $\left(P^{*}=500 \mathrm{~atm}\right.$ for argon and $10^{5} \mathrm{~atm}$ for krypton) and to the pressure $P_{0}=1 \mathrm{~atm}$ at the nozzle entry. In Fig. 4 


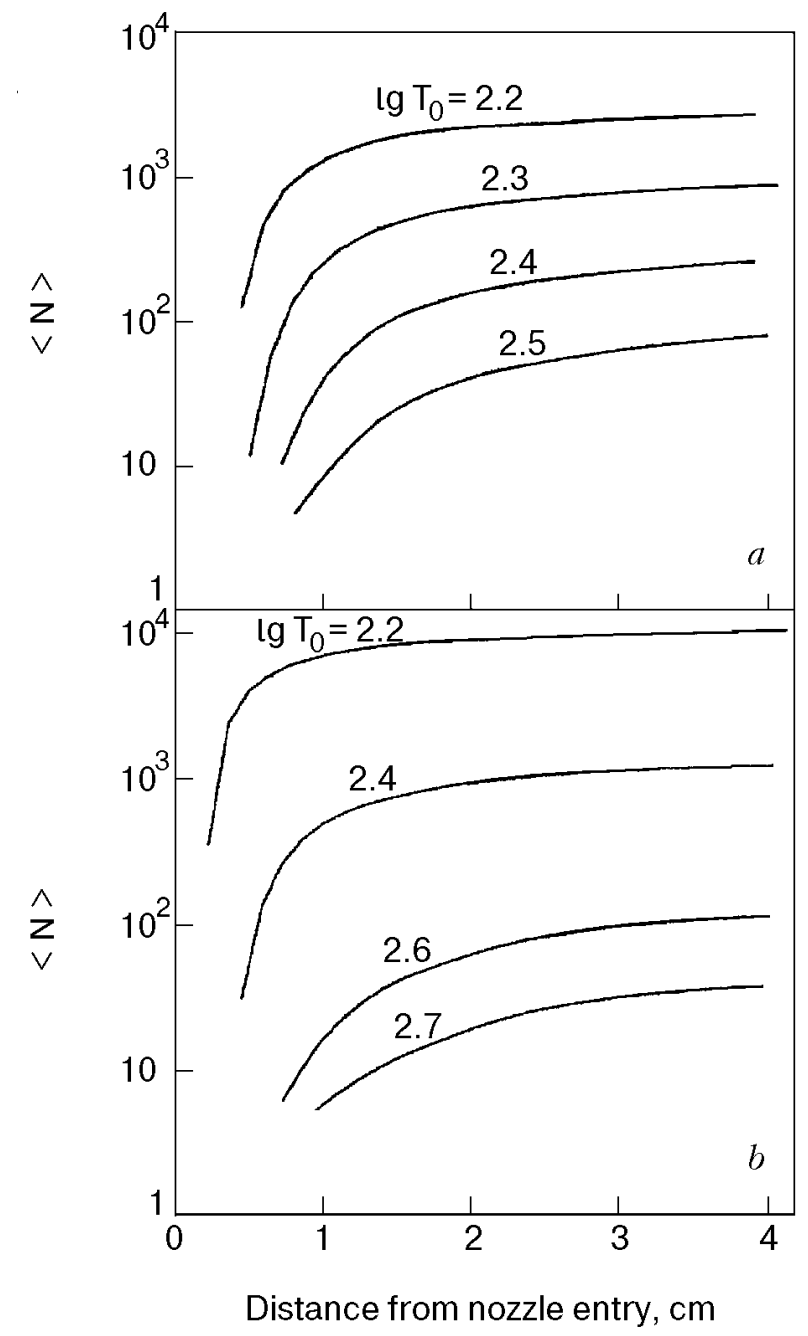

Fig. 4. Mean cluster size, $\langle N\rangle$, versus distance from the nozzle entry for the same initial pressure $P_{0}=1 \mathrm{~atm}$ and different initial temperatures (in Kelvins): argon $(a)$ and krypton $(b)$.

mean cluster size is presented versus distance, $x$, from the nozzle entry for different fixed temperatures at the nozzle entry. It is interesting to compare these data with Fig. 5 which shows the relative fraction of clusters in the total mass of the substance as a function of the same variable $x$. As can be seen from Fig. 5, at the distance $x=1$ to $4 \mathrm{~cm}$ the mass of clusters achieves saturation (gas condensation is blocked due to a growth of gas entropy and the corresponding lowering of $T_{c}$ demonstrated by Fig. 1). But in the region of mass saturation, as is obvious from Fig. 4, the mean cluster size continues to grow due to cluster-cluster aggregation (the slopes of the curves in Figs. 4 and 5 should be compared with the allowance for different-type scales on the ordinate axes). This indicates an essential contribution of the cluster-cluster aggregation process to the formation of the size distribution of clusters.

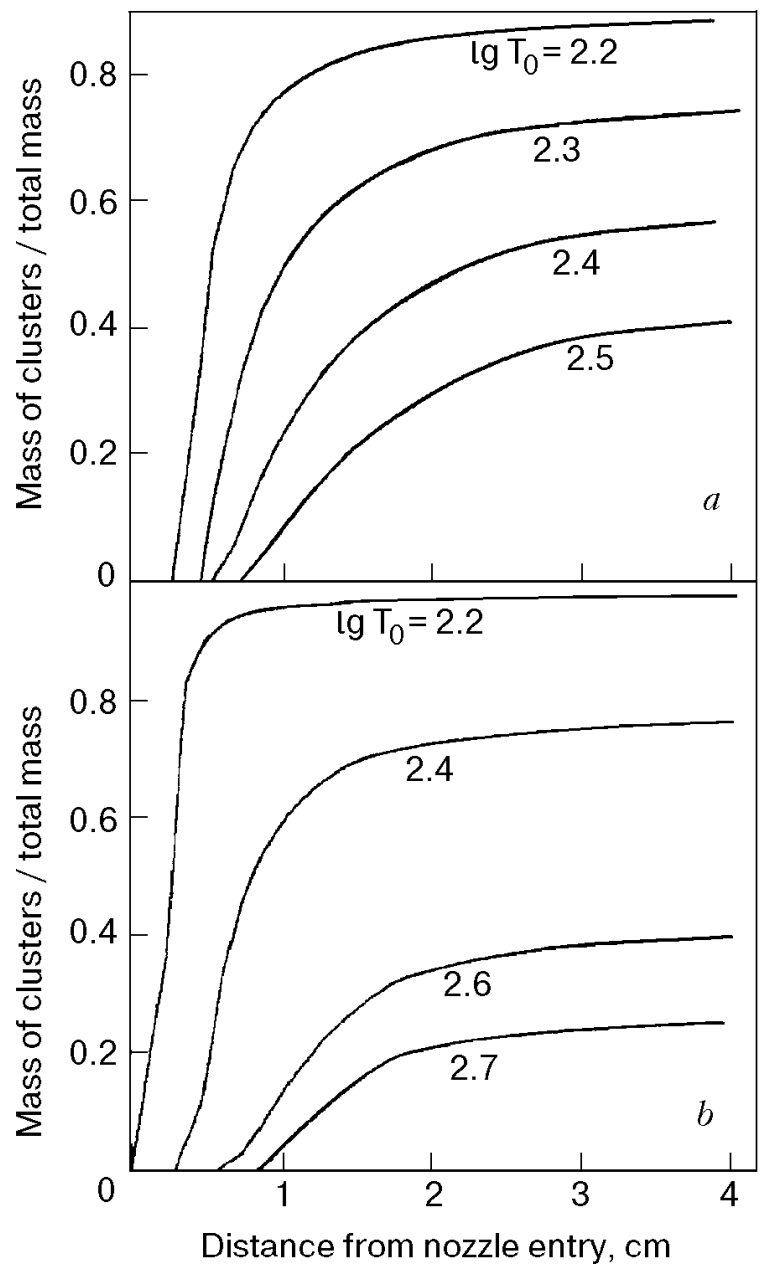

Fig. 5. The fraction of clusters in the total mass of the substance versus distance from the nozzle entry for the same initial pressure $P_{0}=1 \mathrm{~atm}$ and different initial temperatures (in Kelvins): argon $(a)$ and krypton $(b)$.

Another evidence for a dominant role of the aggregation mechanism in the formation of the size distribution function (25) is provided by the form of the distribution function. As a result of calculations, it was found that the function, $f(n)$, of the cluster mass distribution over relative cluster size $n=N /\langle N\rangle$ is well enough approximated in the simple form

$$
f(n) \equiv F(n\langle N\rangle)=\text { const } n \exp (-k n)
$$

with a constant multiplier $k$ which takes up values in a narrow interval

$$
1.1 \leq k \leq 1.2
$$

in all the cases examined. The approximation (27) holds in the total range of mean sizes $\langle N\rangle>50$ where the developed method of calculation is applicable. For krypton $k$ is always close to 1.1 ; in the case of argon $k$ is near to 1.1 for $\langle N\rangle \leq 100$ and to 1.2 for $\langle N\rangle \geq 300$. As an example, Fig. 6 presents 

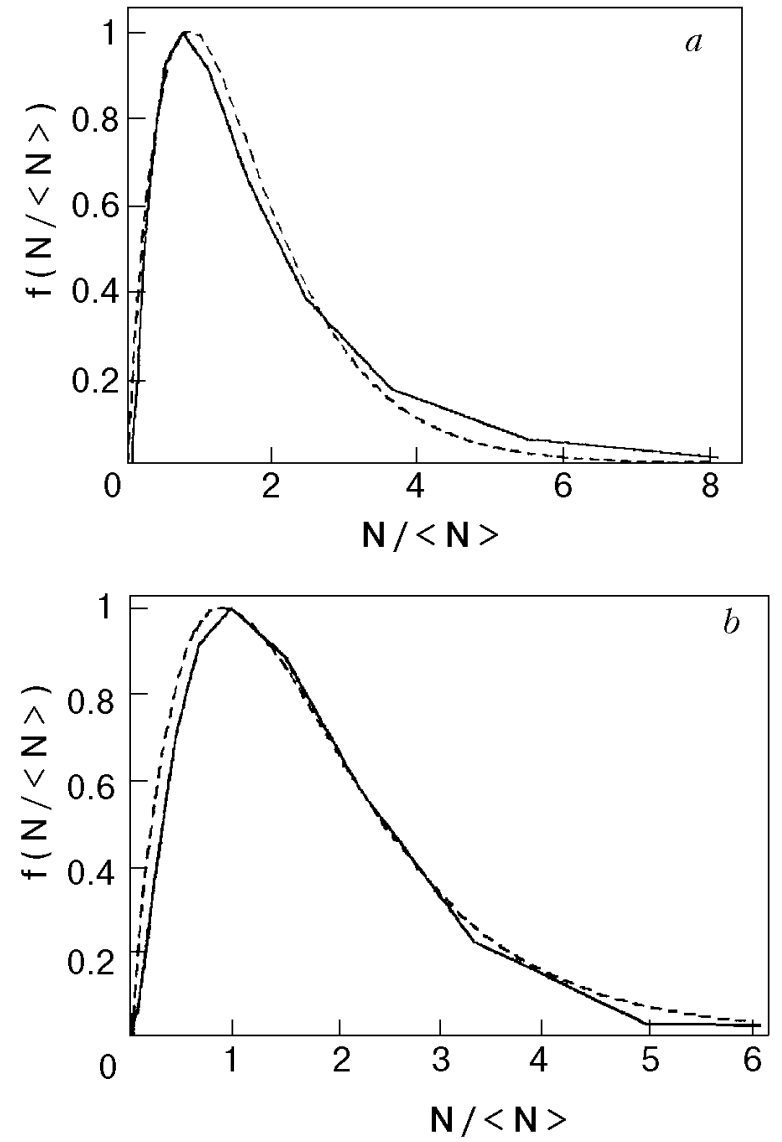

Fig. 6. The function of the cluster mass distribution $f(N /\langle N\rangle)$ over cluster sizes for argon $(a)$ and krypton $(b)$ normalized to the unit value of the maximum ordinate. Solid line - calculation results, dashed line - approximation (27) with $k=1.2$ for argon and 1.1 for krypton. The figure relates to $P_{0}=1 \mathrm{~atm}$, $\lg T_{0}(K)=2.4$ and the $4.1 \mathrm{~cm}$ distance from the nozzle entry.

the distribution function, calculated for the initial conditions $P_{0}=1 \mathrm{~atm}, \lg T_{0}=2.4$ (solid line) and its approximation (27) with $k=1.2$ for argon and 1.1 for krypton (dashed line).

The distribution function (27) is formed mainly in the process of cluster-cluster aggregation. Indeed, the calculations show that in the absence of gas condensation the aggregation process results in the formation of the size distribution function (27) with $k=1$. A slight deviation of the coefficient $k$ from unity is connected with the condensation growth of clusters: the condensation flux of atoms onto the cluster surface, being proportional to $N^{2 / 3}$, grows with cluster size $N$ slower than $N$ which results in a slight shortening of the large-size tail of the distribution function. This is allowed for by the coefficient $k>1$ in Eq. (27).

The size distribution function (27) also gives the distribution of clusters over intrinsic temperature which is maintained close to $T_{c}$ in the course of cluster growth ( $T_{c}$ as a function of the gas state and cluster size $N$ is given by Eqs. (2)-(9)] .

It should be noted in conclusion that the developed approach explains the physical meaning of an important experimental regularity (usually called the similarity law): all the properties of the jet related to clusters depend on a combination of the nozzle-entry parameters $P_{0}^{-1} T_{0}^{n}$ with $n$ close to $5 / 2$ [22]. The point is that the cluster growth process is mainly determined by gas entropy per atom, $s_{g}$, which dictates the cluster temperature in the course of condensation growth (see Sec. 2). It is just the variable $P_{0}^{-1} T_{0}^{5 / 2}$ on which the initial entropy, $s_{g}$, depends according to Eq. (4).

The author is deeply grateful to Dr. E. T. Verkhovtseva for helpful discussions.

This work was carried out within the project of Ukrainian scientific Techological Center № 593

1. Proc. 4th Int. Symp. of Small Particles, Inorganic Clusters, Z. Phys. D12 (1990).

2. J. Stapelfeld, J. Wormer, and T. Moller, Phys. Rev. Lett. 62, 98 (1989).

3. J. Wormer, V. Guzielaski, J. Stapelfeld, and T. Moller, Chem. Phys. Lett. 159, 321 (1989).

4. J. Wormer, V. Guzielaski, J. Stapelfeld, G. Zimmerer, and T. Moller, Phys. Scripta 41, 490 (1990).

5. J. Wormer, M. Joppien, G. Zimmerer, and T. Moller, Phys. Rev. Lett. 67, 2053 (1991).

6. E. T. Verkhovtseva, E. A. Bondarenko, and Yu. S Doronin, Chem. Phys Lett. 140, 181 (1987).

7. E. A. Bondarenko, E. T. Verkhovtseva, Yu. S. Doronin, and M. A. Ratner, Chem. Phys. Lett. 182, 637 (1991).

8. E. T. Verkhovtseva, E. A. Bondarenko, and Yu. S. Doronin, Khim. Fizika 10, 956 (1991).

9. S. S. Kovalenko, D. D. Solnyshkin, E. A. Bondarenko, and E. T. Verkhovtseva, Fiz. Nizk. Temp. 23, 190 (1997) [Low Temp. Phys.] 23, 140 (1997).

10. K. Kobashi and R. D. Etters, Surf. Sci. 150, 252 (1985).

11. S. C. Wang, Phys. Rev. Lett. 75, 2496 (1995).

12. V. G. Karpov, Phys. Rev. Lett. 75, 2702 (1995).

13. D. I. Zhukhovitski, JETP 109, 839 (1996).

14. E. A. Bondarenko, E. T. Verkhovtseva, A. K. Kupko, and T. S. Chebanova, Fiz. Nizk. Temp. 6, 376 (1980) [Sov. J. Low Temp. Phys 6, 180 (1980)].

15. R. Thouy, and R. Jullien, J. Phys. A27, 2954 (1994).

16. E. Ben Naim, P. L. Krapivski, and S. Redner, Phys. Rev. E50, 822 (1994).

17. U. Buck and R. Krohne, Phys. Rev. Lett. 73, 947 (1994).

18. R. Schmidt, G. Seifert, and H. O. Lutz, Phys. Lett. A158, 231 (1991).

19. O. Knospe, R. Schmidt, E. Engel, U. R. Schmitt, R. M. Dreizler, and H. O. Lutz, Phys. Lett. A183, 332 (1993).

20. R. Schmidt and H. O. Lutz, Phys. Lett. A183, 338 (1993).

21. A. M. Ratner and O. Ya. Shamfarova, Fiz. Nizk. Temp. 2, 1551 (1976) [Sov. J. Low Temp. Phys. 2, 756 (1976)].

22. O. F. Hagena and W. Obert, J. Chem. Phys. 56, 1793 (1972). 